\title{
Evaluación de la restrictividad de dispositivos residenciales para personas con un diagnóstico psiquiátrico en el modelo de Salud Mental Comunitaria en Chile*
}

\author{
Assessment of the Restrictiveness of Supported Housing \\ Services for People with Psychiatric Diagnosis in the \\ Model of Community Mental Health in Chile
}

Recibido: $1^{\circ}$ de marzo de 2015 | Revisado: $1^{\circ}$ de junio de 2015 | Aceptado: $1^{\circ}$ de agosto de 2015

\author{
Pamela Grandón FernándeZ ** \\ Claudio Bustos Navarrete \\ FÉlIX COVA SOlAR \\ Universidad de Concepción, Chile \\ Carolina Tapia CÁRdenas \\ Servicio de Salud Concepción, Chile
}

doi:10.11144/Javeriana.upsy14-4.erdr

Para citar este artículo: Grandón, P., Bustos, C., Cova, F., \& Tapia, C. (2015). Evaluación de la restrictividad de dispositivos residenciales para personas con un diagnóstico psiquiátrico en el modelo de salud mental comunitaria en Chile. Universitas Psychologica, 14(4), 1359-1370. http:/ dx.doi.org/10.11144/Javeriana.upsy14-4.epbs

Artículo sin financiación

*** Universidad de Concepción. pgrandon@udec.cl Universidad de Concepción. clbustos@udec.cl

Universidad de Concepción. fecova@udec.cl

Servicio de Salud Concepción. catapia1@puc.cl

\section{RES UMEN}

Los hogares y residencias protegidas son elementos centrales de la red asistencial que atiende a personas con un diagnóstico de trastorno mental severo, dentro del modelo de salud mental comunitaria. Este modelo tiene como uno de sus propósitos centrales estimular la autonomía y participación social de los usuarios. Se evaluó la restrictividad presente en estos dispositivos y factores asociados a su predicción. Se evaluaron 21 servicios residenciales mediante la escala de prácticas restrictivas, que se aplicó a un cuidador por lugar. Se observó que estos dispositivos poseen un nivel de restrictividad que limita la capacidad de los residentes para tomar decisiones en aspectos de su vida. Esta situación dificulta el empoderamiento y la participación de las personas en la comunidad.

Palabras clave

salud mental comunitaria; restrictividad; servicios residenciales; trastornos mentales graves

\footnotetext{
A B S T R A C T

Supported housing services are key elements of the healthcare network that serves people with a diagnosis of severe mental illness, within the community mental health model. This model has as a central purpose to stimulate both autonomy and social participation of users. The restrictiveness present in these centers and factors associated to their prediction was assessed. A total of 21 residential services were assessed by means of the scale of restrictive practices applied to one caregiver per place. It was observed that these services have a level of restrictiveness that limits the ability to make decisions of the residents respect to their lives. This situation hinders both empowerment and participation of people in their communities.

Keywords

community mental health; restrictiveness; supported housing services; severe mental illness
} 
Las personas con un diagnóstico de Trastorno Mental Severo (TMS) han sufrido una larga historia de marginación. Hasta hace pocas décadas, el principal modelo de atención a las necesidades y problemas de salud mental y psicosociales de esta población se organizaba en torno a la internación en instituciones psiquiátricas durante largos períodos de tiempo o incluso de por vida. Sin embargo, desde hace algunos años, la Organización Mundial de la Salud ha recomendado un modelo de atención comunitario para esta población (Organización Mundial de la Salud, 2006; Organización Panamericana de la Salud, 1991). La salud mental comunitaria (SMC) es un modelo que trasciende la mera asistencia clínica, centrada en el usuario como asiento individual de sufrimiento, para proyectarse en la comunidad; es allí donde surge el malestar del sujeto, y por tanto, donde puede ocurrir la recuperación del mismo (Marcos \& Topa, 2012). Implica el reconocimiento de la comunidad no solo como usuaria, sino también como generadora de recursos que deben aliarse con los específicamente técnicos para hacer frente a sus problemas de salud. Este modelo, al igual que la psicología comunitaria, fomenta la participación y el empoderamiento de las personas (Cantera, 2004; Perron, Rudge, \& Holmes, 2010; Storm \& Edwards, 2013). Solo será posible la recuperación de los sujetos si estos vuelven a formar parte del tejido social, del que han sido excluidos, a través del ejercicio ciudadano (Saraceno, 2003). Sobre todo considerando el fuerte estigma que pesa sobre las personas que presentan un diagnóstico psiquiátrico severo, diversas investigaciones señalan que los estereotipos sociales las presentan como peligrosas, irracionales e incapaces de cuidarse; creencias que también poseen los equipos de salud (Liggins \& Hatcher, 2005; Rose, Thornicroft, Pinfold, \& Kassam, 2007). Estas actitudes inciden en la intervención que se realiza, pues se justifica como necesario un trato autoritario e infantilizador que perpetúa la exclusión y el estigma (Corrigan, 2002).

Desde lo práctico, la SMC pasa por una forma de organizar la asistencia psiquiátrica que se caracteriza por incluir programas enfocados hacia la promoción de la salud mental, prevención y tratamiento de los trastornos mentales, y rehabilitación e inclusión en la comunidad (Ministerio de Salud, 2008).

El Ministerio de Salud de Chile, a través del "Plan Nacional de Salud Mental y Psiquiatría", adhiere a este modelo y a partir de él ha desarrollado una red de dispositivos de atención comunitaria destinados a estos propósitos (Ministerio de Salud, 2001). Un desafío para este modelo, en el área de la rehabilitación e inclusión social, es el diseño de estructuras residenciales que eviten la replicación de modelos masificados y reproductores de la institucionalización. En este contexto, surgen los hogares y residencias protegidas entendidos también como "dispositivos de apoyo social", que tratan de contrarrestar las dificultades y problemas derivados del sufrimiento mental y de eliminar algunas de las barreras que bloquean el pleno funcionamiento ciudadano de las personas con un diagnóstico de TMS (López \& Laviana, 2007).

Los hogares y residencias protegidas pretenden favorecer la inclusión social a través de la integración de estas personas en sus vecindarios (Kloos $\&$ Shah, 2009). Para ello se trabaja favoreciendo la autonomía personal, bajo el supuesto que a través del ejercicio de recuperación del rol social, las personas pueden empoderarse y hacerse valer como sujetos de derechos. En este sentido, estos dispositivos deberían fomentar la participación de los usuarios en su organización, mediante la toma de decisiones sobre todos aquellos aspectos que los afecten (Ministerio de Salud, 2000). Sin embargo, no siempre es así; existen modelos de organización de las viviendas que facilitan la recuperación e integración y otros que se mantienen bajo una perspectiva más asilar (Wright \& Kloos, 2007). En esta línea, algunas investigaciones señalan que el nivel de restrictividad del dispositivo se asocia negativamente con la autonomía que desarrollan los usuarios, además de influir en la relación que se establece entre los cuidadores y los residentes (Kyle \& Dunn, 2008; López, Fernández, García-Cubillana, Moreno, Jimeno, \& Laviana, 2005). Sprioli y Silva (2011) encontraron que, en un ambiente no estructurado, el personal se relacionaba con los usuarios con mayor normalidad, en tanto en un ambiente altamente estructurado se comportaba de una for- 
ma más profesional y respondía en función de la percepción que tenía el usuario del funcionamiento. En este sentido, se ha encontrado que la restrictividad se asocia con las habilidades domésticas y comunitarias de residentes de servicios residenciales; específicamente, una menor restrictividad del centro se relaciona con mayores habilidades para la vida diaria en los usuarios (Tapia, 2014).

En relación con lo expuesto y considerando la escasa investigación que existe sobre la implementación práctica del modelo de SMC tanto en Chile como en otros países de Latinoamérica, esta investigación tuvo como propósito conocer cómo el modelo de SMC se operacionaliza en los servicios residenciales. Específicamente, el objetivo del estudio fue evaluar el nivel de restrictividad de dispositivos residenciales e identificar variables, dependientes del área sanitaria y del dispositivo, que están implicadas en la predicción de esta. La hipótesis del estudio es que características del área sanitaria y del dispositivo contribuirán a la predicción de los niveles de restrictividad de los servicios residenciales.

\section{Método}

La investigación realizada es de tipo descriptivocorrelacional. Las medidas fueron obtenidas en solo un solo corte de tiempo (diseño transversal).

\section{Participantes}

El estudio se realizó en 15 hogares y 6 residencias protegidas ubicados en las regiones del Maule y Biobío de Chile. Los dispositivos pertenecían a las áreas sanitarias de Maule, Nuble, Biobío, Talcahuano, Concepción y Arauco. Solo se incluyeron los hogares con un tiempo de funcionamiento mayor a un año y las residencias que funcionaran más de ocho meses. La información sobre las características generales del centro y sus prácticas restrictivas se obtuvo de un cuidador por lugar, a excepción de la pregunta sobre la homogeneidad en el funcionamiento de los dispositivos residenciales del área sanitaria, que se le hizo a la persona encargada de salud mental de toda el área de salud respectiva.
El cuidador se seleccionó por conveniencia entre aquellos trabajadores de los hogares y residencias que laboraran hace más de un año en ellos. Por tanto, se entrevistó a 21 cuidadores de los cuales el $93.3 \%$ fueron mujeres.

\section{Instrumentos}

\section{Escala de Prácticas Restrictivas (Jordá \& Espinosa, 1990)}

Evalúa el funcionamiento de los dispositivos residenciales en las siguientes áreas: actividades personales, posesiones personales, comida, higiene y salud, habitaciones de los residentes y servicios. El instrumento original consta de 55 preguntas dicotómicas ( $\mathrm{Sí} / \mathrm{No}$ ) relacionadas con las áreas enumeradas. El estudio de fiabilidad del instrumento se realizó en 58 alojamientos situados en la provincia de Valencia, donde habían sido trasladados personas con TMS (Jordá \& Espinosa, 1990). Se corroboró la fiabilidad test-retest entre entrevistadores, obteniéndose un coeficiente rho (Spearman) = 0.98 significativo $p<0.05$. La correlación biserial entre cada ítem y la puntuación total de la escala estuvo en un rango entre 0.2 y 0.97. Para su empleo en este estudio, se eliminaron 12 ítems que no eran aplicables en la realidad del país, se cambiaron a positivo todas las preguntas redactadas en sentido inverso en el original y se utilizó un formato de respuesta tipo Likert con cuatro alternativas: siempre, generalmente, rara vez y nunca. El instrumento final quedó conformado por 43 ítems. Se obtuvo un coeficiente de confiabilidad alfa de Cronbach de 0.82 para la escala.

Además se elaboró una ficha para la recolección de información del cuidador y del dispositivo. Respecto al hogar o residencia, se consultó por: (a) la frecuencia de asesoría técnica, (b) la asignación de tareas y horarios de forma personalizada a cada residente, (c) la homogeneidad de funcionamiento de los dispositivos dentro del área sanitaria respectiva, (d) el tipo de profesional que realiza la asesoría técnica (e) el tipo de dispositivo (hogar, residencia) y (f) el tiempo de funcionamiento. 


\section{Procedimiento}

Se aplicó una prueba piloto en una residencia protegida que no fue incorporada en el estudio definitivo. Posteriormente, se revisó el instrumento para readecuarlo al contexto local. Se tomó contacto con los encargados de salud mental de todas las áreas sanitarias incluidas. Se les consultó sobre la cantidad de dispositivos residenciales de su área, la homogeneidad en su funcionamiento y su disponibilidad para colaborar con la investigación. Una vez obtenida la autorización de los comités de éticas de los distintos lugares, se iniciaron las visitas a los dispositivos respectivos.

Se realizó una entrevista al cuidador que, cumpliendo con los criterios de inclusión, se encontrara presente en el momento de la visita. Se les explicó el objetivo del estudio y se solicitó su colaboración. A quienes accedieron a participar y firmaron el consentimiento informado se les aplicó individualmente los instrumentos, en dependencias del mismo establecimiento.

\section{Análisis de los datos}

Se ingresaron los datos al sistema estadístico SPSS versión 15 y se analizaron las respuestas utilizando el software estadístico $R$ versión 3.1.2. Se estableció la consistencia interna de la escala de prácticas restrictivas usando el alfa de Cronbach.

En primer lugar, se estimaron los estadísticos descriptivos para la restrictividad global de los dispositivos. Para evaluar la restrictividad de las prácticas específicas, se separaron las prácticas restrictivas del dispositivo en generales y aquellas que tratan de restricciones hacia los usuarios. Se utilizó la prueba de Friedman para determinar si existían diferencias en la restrictividad de las prácticas, independientemente del lugar. Finalmente, se realizó una regresión múltiple por mínimos cuadrados ordinarios para identificar las variables del área sanitaria y del dispositivo, que permiten predecir el nivel de restrictividad global.

\section{Resultados}

La media de restrictividad global de los centros es de $2.63(D E=0.34)$, lo que indica que se encuentra sobre el punto medio teórico de 2.5. La distribución es casi simétrica (asimetría $=0.03$ ) y con una leve curtosis negativa (curtosis $=-0.79$ ), lo que nos permite afirmar que la distribución de los datos es prácticamente normal, que se confirma al utilizar la prueba de Shapiro-Wilk: $\mathrm{W}=0.977, p=0.877$.

$\mathrm{Al}$ analizar las prácticas específicas presentes en la escala de restrictividad, 23 de los 43 ítems miden restricciones generales, en tanto que 20 representan restricciones directas hacia el residente.

Usando la prueba de Friedman, se observaron diferencias estadísticamente significativas en el nivel de restrictividad general, $\chi_{(22)}^{2}=175.49, p<$ 0.001 . Como se puede ver en la Tabla 1 , las mayores restricciones de los dispositivos están en los horarios, tanto de llegada al lugar como de ir acostarse durante los días de semana. En relación con este último punto, se vigila que los residentes estén en sus camas durante la noche. También el personal puede ingresar a las habitaciones en cualquier momento, maneja los fármacos de los usuarios y en un $80 \%$ de los dispositivos entre, siempre y generalmente, el dinero de los residentes es administrado por los cuidadores. En términos de las actividades donde existen menores restricciones, se encuentra el permitir visitas a los residentes, la ausencia de visitas por parte de psiquiatra al dispositivo, el no control del peso de los residentes y la libertad para decorar las habitaciones.

$\mathrm{Al}$ analizar la restrictividad directa hacia los residentes, existen diferencias estadísticamente significativas entre las distintas prácticas, independientemente del dispositivo, $\chi_{(19)}^{2}=229.35, p$ $<0.001$. En la Tabla 2 se puede ver que las tres conductas que no se permiten en ningún hogar son: fumar en las habitaciones, beber bebidas alcohólicas y salir en la noche sin informar al personal. En un $81 \%$ de los lugares no se puede cerrar la puerta del baño con llave, y en un $76.2 \%$ no se les permite prepararse alimentos fuera de horario ni tener llave de la puerta principal. Por otra parte, los residentes tienen mayor libertad en aquellas actividades que tienen que ver con la administración de sus pertenecías personales y con la posibilidad de elegir ropa, descanso en su habitación y prepararse una bebida caliente. 
TABLA 1

Distribución de prácticas restrictivas generales

\begin{tabular}{|c|c|c|c|c|c|c|c|c|c|c|c|}
\hline \multirow{2}{*}{ Ítem } & \multirow{2}{*}{ Descripción } & \multicolumn{2}{|c|}{ Nunca } & \multicolumn{2}{|c|}{ Rara vez } & \multicolumn{2}{|c|}{ Generalmente } & \multicolumn{2}{|c|}{ Siempre } & \multirow[t]{2}{*}{ Mediana } & \multirow[t]{2}{*}{ Media } \\
\hline & & $n$ & $\%$ & $n$ & $\%$ & $N$ & $\%$ & $n$ & $\%$ & & \\
\hline 13 & $\begin{array}{l}\text { Durante la semana, los residentes deben } \\
\text { regresar antes de las 23:00 h. }\end{array}$ & 0 & 0 & 0 & 0 & 0 & 0 & 21 & 100 & 4 & 4 \\
\hline 18 & $\begin{array}{l}\text { Durante la noche el personal revisa que los } \\
\text { residentes estén en la cama. }\end{array}$ & 0 & 0 & 0 & 0 & 1 & 4.8 & 20 & 95.2 & 4 & 4 \\
\hline 11 & $\begin{array}{l}\text { La puerta principal permanece cerrada a } \\
\text { partir de las 22:00 } \mathrm{h} \text {. }\end{array}$ & 1 & 4.8 & 0 & 0 & 0 & 0 & 20 & 95.2 & 4 & 3.9 \\
\hline 29 & $\begin{array}{l}\text { Los fármacos de los usuarios son } \\
\text { manejados por el personal. }\end{array}$ & 1 & 4.8 & 0 & 0 & 0 & 0 & 20 & 95.2 & 4 & 3.9 \\
\hline 4 & $\begin{array}{l}\text { El personal de la residencia/hogar puede } \\
\text { entrar en las habitaciones a cualquier hora. }\end{array}$ & 2 & 9.5 & 0 & 0 & 0 & 0 & 19 & 90.5 & 4 & 3.7 \\
\hline 9 & $\begin{array}{l}\text { Los residentes deben preguntar al personal } \\
\text { cuando quieren asistir a médico general. }\end{array}$ & 2 & 9.5 & 0 & 0 & 2 & 9.5 & 17 & 81.0 & 4 & 3.6 \\
\hline 14 & $\begin{array}{l}\text { Durante la semana se recomienda que los } \\
\text { residentes estén acostados antes de las } \\
\text { 23:00 h. }\end{array}$ & 3 & 14.3 & 0 & 0 & 1 & 4.8 & 17 & 81.0 & 4 & 3.5 \\
\hline 25 & $\begin{array}{l}\text { El personal es responsable del dinero de los } \\
\text { residentes. }\end{array}$ & 1 & 4.8 & 3 & 14.3 & 5 & 23.8 & 12 & 57.1 & 4 & 3.3 \\
\hline 1 & $\begin{array}{l}\text { Los residentes deben levantarse } \\
\text { diariamente a una hora determinada. }\end{array}$ & 1 & 4.8 & 1 & 4.8 & 9 & 42.9 & 10 & 47.6 & 3 & 3.3 \\
\hline 10 & $\begin{array}{l}\text { Hay reuniones periódicas entre el personal } \\
\text { y los residentes. }\end{array}$ & 5 & 23.8 & 1 & 4.8 & 4 & 19 & 11 & 52.4 & 4 & 3 \\
\hline 40 & El baño está supervisado por el personal. & 6 & 28.6 & 2 & 9.5 & 0 & 0 & 13 & 61.9 & 4 & 3 \\
\hline 16 & $\begin{array}{l}\text { Los fines de semana se recomienda } \\
\text { acostarse a las } 23: 00 \mathrm{~h} .\end{array}$ & 4 & 19 & 2 & 9.5 & 6 & 28.6 & 9 & 42.9 & 3 & 3 \\
\hline 17 & $\begin{array}{l}\text { Los residentes deben estar acostados a una } \\
\text { hora determinada. }\end{array}$ & 5 & 23.8 & 2 & 9.5 & 4 & 19 & 10 & 47.6 & 3 & 2.9 \\
\hline 35 & $\begin{array}{l}\text { Periódicamente se revisan las pertenencias } \\
\text { personales de los residentes para buscar } \\
\text { cosas prohibidas. }\end{array}$ & 5 & 23.8 & 6 & 28.6 & 0 & 0 & 10 & 47.6 & 2 & 2.7 \\
\hline 2 & $\begin{array}{l}\text { Durante los fines de semana los residentes } \\
\text { deben levantarse a una hora determinada. }\end{array}$ & 5 & 23.8 & 4 & 19 & 6 & 28.6 & 6 & 28.6 & 3 & 2.6 \\
\hline 3 & $\begin{array}{l}\text { Las puertas de la residencia/hogar deben } \\
\text { permanecer cerradas para evitar que los } \\
\text { residentes salgan. }\end{array}$ & 8 & 38.1 & 3 & 14.3 & 3 & 14.3 & 7 & 33.3 & 2 & 2.4 \\
\hline 43 & $\begin{array}{l}\text { Las pertenencias personales de los } \\
\text { residentes están catalogadas. }\end{array}$ & 10 & 47.6 & 1 & 4.8 & 3 & 14.3 & 7 & 33.3 & 2 & 2.3 \\
\hline 31 & $\begin{array}{l}\text { Hay un control periódico de peso de los } \\
\text { residentes. }\end{array}$ & 12 & 57.1 & 1 & 4.8 & 1 & 4.8 & 7 & 33.3 & 1 & 2.1 \\
\hline 20 & $\begin{array}{l}\text { Un peluquero visita regularmente la } \\
\text { residencia/hogar. }\end{array}$ & 14 & 66.7 & 1 & 4.8 & 1 & 4.8 & 5 & 23.8 & 1 & 1.9 \\
\hline 30 & $\begin{array}{l}\text { En el momento de la llegada de los } \\
\text { usuarios a la residencia/hogar, estos deben } \\
\text { ser pesados. }\end{array}$ & 14 & 66.7 & 1 & 4.8 & 1 & 4.8 & 5 & 23.8 & 1 & 1.9 \\
\hline 8 & $\begin{array}{l}\text { Hay un psiquiatra que visita regularmente } \\
\text { la residencia/hogar. }\end{array}$ & 14 & 66.7 & 2 & 9.5 & 1 & 4.8 & 4 & 19.0 & 1 & 1.8 \\
\hline 6 & $\begin{array}{l}\text { El horario de visitas a los residentes está } \\
\text { restringido. }\end{array}$ & 14 & 66.7 & 3 & 14.3 & 2 & 9.5 & 2 & 9.5 & 1 & 1.6 \\
\hline 34 & $\begin{array}{l}\text { Hay restricciones a la libre decoración de } \\
\text { espacios personales (habitaciones). }\end{array}$ & 15 & 71.4 & 3 & 14.3 & 0 & 0 & 3 & 14.3 & 1 & 1.6 \\
\hline
\end{tabular}

Nota. Valor más alto de la media y mediana indica mayor nivel de restricción.

Fuente: elaboración propia 
TABLA 2

Distribución de la restrictividad directa hacia los residentes

\begin{tabular}{|c|c|c|c|c|c|c|c|c|c|c|c|}
\hline \multirow[t]{2}{*}{ Ítem } & \multirow[t]{2}{*}{ Descripción } & \multicolumn{2}{|c|}{ Siempre } & \multicolumn{2}{|c|}{$\begin{array}{l}\text { General- } \\
\text { mente }\end{array}$} & \multicolumn{2}{|c|}{ Rara vez } & \multicolumn{2}{|c|}{ Nunca } & \multirow[t]{2}{*}{ Mediana } & \multirow[t]{2}{*}{ Media } \\
\hline & & $n$ & $\%$ & $n$ & $\%$ & $n$ & $\%$ & $n$ & $\%$ & & \\
\hline 15 & $\begin{array}{l}\text { Los residentes pueden salir solos } \\
\text { por la noche sin informar al per- } \\
\text { sonal. }\end{array}$ & 0 & 0 & 0 & 0 & 0 & 0 & 21 & 100 & 4 & 4 \\
\hline 24 & $\begin{array}{l}\text { Está permitido el consumo de } \\
\text { bebidas alcohólicas. }\end{array}$ & 0 & 0 & 0 & 0 & 0 & 0 & 21 & 100 & 4 & 4 \\
\hline 42 & $\begin{array}{l}\text { Los residentes pueden fumar en } \\
\text { las habitaciones. }\end{array}$ & 0 & 0 & 0 & 0 & 0 & 0 & 21 & 100 & 4 & 4 \\
\hline 23 & $\begin{array}{l}\text { Los residentes pueden prepararse } \\
\text { comida fuera de los horarios esta- } \\
\text { blecidos. }\end{array}$ & 2 & 9.5 & 0 & 0 & 3 & 14.3 & 16 & 76.2 & 4 & 3.6 \\
\hline 12 & $\begin{array}{l}\text { Los residentes tienen llave de la } \\
\text { puerta principal. }\end{array}$ & 2 & 9.5 & 2 & 9.5 & 1 & 4.8 & 16 & 76.2 & 4 & 3.5 \\
\hline 39 & $\begin{array}{l}\text { Los residentes pueden cerrarse } \\
\text { con llave en el WC. }\end{array}$ & 4 & 19 & 0 & 0 & 0 & 0 & 17 & 81 & 4 & 3.4 \\
\hline 5 & $\begin{array}{l}\text { Los residentes pueden ver TV } \\
\text { después de las 23:00 h. }\end{array}$ & 4 & 19 & 0 & 0 & 6 & 28.6 & 11 & 52.4 & 4 & 3.1 \\
\hline 7 & $\begin{array}{l}\text { En la residencia/hogar está per- } \\
\text { mitido que las personas de sexo } \\
\text { contrario (excluido el personal) } \\
\text { entren en las habitaciones. }\end{array}$ & 4 & 19 & 1 & 4.8 & 4 & 19 & 12 & 57.1 & 4 & 3.1 \\
\hline 21 & $\begin{array}{l}\text { Los residentes participan en la } \\
\text { planificación de la alimentación. }\end{array}$ & 2 & 9.5 & 6 & 28.6 & 5 & 23.8 & 8 & 38.1 & 3 & 2.9 \\
\hline 27 & $\begin{array}{l}\text { Está permitido que los residentes } \\
\text { tengan máquinas de afeitar, cu- } \\
\text { chillos o tijeras. }\end{array}$ & 6 & 28.6 & 0 & 0 & 7 & 33.3 & 8 & 38.1 & 3 & 2.8 \\
\hline 37 & $\begin{array}{l}\text { Los residentes se lavan sus propias } \\
\text { ropas. }\end{array}$ & 7 & 33.3 & 2 & 9.5 & 7 & 33.3 & 5 & 23.8 & 3 & 2.5 \\
\hline 26 & $\begin{array}{l}\text { Está permitido que los residentes } \\
\text { tengan fósforos o encendedores. }\end{array}$ & 8 & 38.1 & 6 & 28.6 & 1 & 4.8 & 6 & 28.6 & 2 & 2.2 \\
\hline 28 & $\begin{array}{l}\text { Los residentes tienen la opción } \\
\text { de guardar bajo llave sus perte- } \\
\text { nencias. }\end{array}$ & 13 & 61.9 & 0 & 0 & 1 & 4.8 & 7 & 33.3 & 1 & 2.1 \\
\hline 41 & $\begin{array}{l}\text { Los residentes pueden elegir la } \\
\text { hora del baño o la ducha. }\end{array}$ & 10 & 47.6 & 5 & 23.8 & 1 & 4.8 & 5 & 23.8 & 2 & 2 \\
\hline 22 & $\begin{array}{l}\text { Los residentes pueden prepararse } \\
\text { un té o café. }\end{array}$ & 14 & 66.7 & 3 & 14.3 & 1 & 4.8 & 3 & 14.3 & 1 & 1.7 \\
\hline 19 & $\begin{array}{l}\text { Los residentes hacen su propia } \\
\text { cama. }\end{array}$ & 17 & 81 & 3 & 14.3 & 0 & 0 & 1 & 4.8 & 1 & 1.3 \\
\hline 36 & $\begin{array}{l}\text { Los residentes pueden descansar } \\
\text { en su cama durante el día. }\end{array}$ & 17 & 81 & 2 & 9.5 & 2 & 9.5 & 0 & 0 & 1 & 1.3 \\
\hline 32 & $\begin{array}{l}\text { Los residentes pueden elegir la } \\
\text { ropa que quieren vestir. }\end{array}$ & 18 & 85.7 & 3 & 14.3 & 0 & 0 & 0 & 0 & 1 & 1.1 \\
\hline 33 & $\begin{array}{l}\text { Los residentes tienen ropa sufi- } \\
\text { ciente. }\end{array}$ & 18 & 85.7 & 3 & 14.3 & 0 & 0 & 0 & 0 & 1 & 1.1 \\
\hline 38 & $\begin{array}{l}\text { Los residentes tienen responsa- } \\
\text { bilidad total sobre el uso de sus } \\
\text { pertenencias. }\end{array}$ & 19 & 90.5 & 2 & 9.5 & 0 & 0 & 0 & 0 & 1 & 1.1 \\
\hline
\end{tabular}

Nota: Valor más alto de la media y mediana indica mayor restrictividad.

Fuente: elaboración propia 


\section{TABLA 3}

Predictores del nivel de restrictividad de los centros

\begin{tabular}{lccccc}
\hline Variable & $\mathrm{B}$ & $\mathrm{EE}$ & $t$ & Valor-p & \\
\hline Constante & -1.17 & 1.01 & -1.16 & 0.27 & \\
Tiempo = entre un año un mes y dos años & -1.59 & 0.58 & -2.73 & 0.02 & $*$ \\
Tiempo = entre dos años un mes y 4 años & -0.66 & 0.97 & -0.68 & 0.51 & \\
Tiempo=mayor a 4 años & 0.07 & 0.82 & 0.08 & 0.93 \\
Regularidad asistencia = una a dos veces por semana & 0.76 & 0.65 & 1.17 & 0.27 \\
Regularidad asistencia = cada 15 días & 0.1 & 0.87 & 0.12 & 0.91 & \\
Regularidad asistencia=cada 3 a 4 meses & 2.98 & 0.97 & 3.08 & 0.01 & $*$ \\
Homogenedad=Sí & 1.84 & 0.51 & 3.64 & 0 & $*$ \\
Tareas personalizadas=Sí & -0.76 & 0.82 & -0.92 & 0.38 & \\
Asistencia=Equipo Servicio de Salud & -0.8 & 0.71 & -1.12 & 0.29 & \\
Tipo dispositivo=residencia & 0.76 & 0.65 & 1.17 & 0.27 & \\
$\mathrm{R}^{2}$ & 0.83 & & & & \\
$\mathrm{~F}$ & 4.879 & & & 0.01 & \\
\hline
\end{tabular}

Nota. Intercepto corresponde al valor estandarizado de la escala de restrictividad para un tiempo de funcionamiento del centro inferior a un año, asistencia técnica diaria por parte del equipo clínico de la red, la ausencia de homogeneidad en las políticas del servicio de salud y de tareas y horarios personalizados, y un dispositivo de tipo hogar.

Fuente: elaboración propia

Para analizar los factores predictivos de la restrictividad global de los centros, se realizó un análisis de regresión múltiple usando como predictores (a) la frecuencia de asesoría técnica, (b) la asignación de tareas y horarios de forma personalizada a cada residente, (c) la homogeneidad de funcionamiento dentro del área sanitaria respectiva, (d) el tipo de profesional que realiza la asesoría técnica (e) el tipo de dispositivo (hogar, residencia) y (f) el tiempo de funcionamiento.

Al analizar el modelo de regresión, en la Tabla 3 se puede observar que este es estadísticamente significativo, con un $83 \%$ de varianza explicada. Las condiciones que permiten predecir la restrictividad global de los centros son (a) el tiempo de funcionamiento, con una menor restrictividad en los centros con un tiempo de funcionamiento entre uno y dos años, (b) la frecuencia de la asistencia técnica: hay presencia de una mayor restrictividad en los centros que tienen una menor regularidad en la asistencia técnica y (c) la homogeneidad de funcionamiento del lugar: existe una mayor restrictividad en los centros cuyas áreas sanitarias son homogéneos en sus políticas.

\section{Discusión}

Los resultados descriptivos indican que los hogares y residencias tienen un mayor nivel de restrictividad global que el punto medio teórico. Al analizar el nivel de restrictividad de las prácticas específicas, generales del dispositivo y del usuario, aparecen diferencias significativas, lo que indica que hay prácticas más restrictivas que otras. Se puede observar que el servicio residencial impone normas rígidas sobre los horarios de llegada, el consumo de alcohol y tabaco y el libre desplazamiento nocturno. Además tienen limitaciones para preparar alimentos y tener llave del lugar. En esta misma línea el personal supervisa que los residentes estén en sus camas durante la noche y tiene la libertad de irrumpir en las habitaciones. Por otra parte, la restrictividad del lugar limita la toma de decisiones de los usuarios en acciones como el manejo de los fármacos y del dinero. Si bien el apoyo hacia los residentes es un aspecto importante en estos dispositivos, este debe estar orientado a que las personas aprendan habilidades y aumenten su autonomía, pues esto favorece su inclusión en la comunidad (Wong \& Solomon, 2002). Este nivel de restrictividad es sorprendente, 
pues en estos lugares cabe imaginar un "ambiente de hogar". En otros países se han encontrado resultados distintos. En Andalucía (España), se evaluaron 16 hogares y 67 viviendas supervisadas, solo una minoría de estos dispositivos tenia normas restrictivas y estaba asociado a ambientes más institucionalizados (López, García-Cubillana, Fernández, Laviana, Maestro, \& Moreno, 2005).

Respecto a la libertad de los residentes, estos tienen la posibilidad de decidir sobre sus objetos personales y en general sobre aspectos que no colocan en juego el control que el dispositivo tiene sobre la estructura y normas del lugar como decorar las habitaciones, elegir la ropa que van a usar o prepararse un té o café.

De acuerdo a estos resultados, la autonomía y capacidad de decidir de los residentes se ve supeditada al dispositivo; aunque estos servicios estén dentro de la red comunitaria de salud mental, continúan reproduciendo prácticas asilares que institucionalizan a los sujetos. La institucionalización de los residentes está inversamente relacionada con la iniciativa y capacidad de participación de estos (López, Lara, Laviana, Fernández, García-Cubillana, \& López, 2004). Desde esta perspectiva, el modelo de SMC al que adhiere la política de salud mental en Chile (Ministerio de Salud, 2001), tiene dificultades para favorecer la participación y el empoderamiento en sus prácticas concretas. Dentro de los principios de la SMC, el empoderamiento es central para que los sujetos puedan pasar de "pacientes" a actores sociales (Corrigan, 2002). Por tanto, los servicios residenciales, al ser un aspecto clave de la red sanitaria, deberían favorecer que los residentes adquieran la capacidad de control de sus vidas y participen en la comunidad (Wong, Filoromo, \& Tennille, 2007).

Algunos autores agregan que además se debe trabajar en procesos de toma de conciencia en este colectivo marginado, para potenciar la adquisición de poder social y político, que les permita organizarse y tener injerencia sobre aspectos de su interés (Kelly, 2006). Para trabajar en este proceso de empoderamiento, los dispositivos residenciales tendrían que fomentar la participación de los usuarios en la toma de decisiones sobre la vida en el hogar, a través de una codirección real de estos servicios con los equipos sanitarios. Esto implica más allá de adoptar la normativa oficial, abrir espacios de negociación entre los distintos actores sociales (Saraceno, 2003). Sin embargo, pese a que la política de salud mental en Chile ha incluido un enfoque comunitario, en la vida cotidiana de los dispositivos residenciales al parecer se continúa considerando a los sujetos como "necesitados de cuidados", con poca capacidad de autorregularse y de decidir sobre aspectos tan básicos como a qué hora dormir.

Esta visión de las personas podría estar relacionada con el estigma que pesa sobre este grupo social. Los estereotipos sociales muestran a las personas con diagnóstico psiquiátrico como peligrosas, irracionales y en general como incapaces de autogestionarse (Rose et al., 2007). Estos prejuicios se actualizan en el trato y la consideración que el dispositivo sanitario tiene por el usuario; alguien peligroso que no puede hacer uso de espacios privados sin supervisión y que requiere la atención de un niño. Desde esta perspectiva, los hogares no han logrado constituirse como contextos empoderadores reales que a su vez propicien procesos de empoderamiento (Silva \& Martínez, 2004). Siguiendo a Montero (2009), el ejercicio desigual del poder entre grupos sociales obstaculiza el empoderamiento, pues naturaliza ciertas formas de exclusión social, que pasan a verse como algo normal e incluso necesario. En este caso, se suele argumentar que las personas con un diagnostico psiquiátrico requieren este tipo restricciones ambientales para poder mejorar; no obstante, tal como lo muestra esta investigación y otras, los distintos perfiles de los residentes no explican los niveles de estructuración de los dispositivos (López, Fernández et al., 2005).

En el presente estudio destaca la gran capacidad explicativa del modelo predictivo empleado $\left(R^{2}=0.83\right)$, en que características relativas al dispositivo y al área sanitaria son las que mayormente contribuyen a determinar el nivel de restrictividad. Estos resultados reafirman que las variables relacionadas con los residentes no son las centrales para determinar la estructura de los dispositivos residenciales. Más bien que esta es- 
taría dada fundamentalmente por la concepción que se tiene de los residentes como sujetos que requieren cuidados permanentes.

Desde esta perspectiva, y de acuerdo a lo encontrado en otros estudios, la promoción de la ciudadanía en los espacios residenciales ha tenido escasa atención (Sylvestre, Nelson, Sabloff, \& Peddle, 2007). Se podría decir que se potencia una ciudadanía "protegida", donde los residentes pueden realizar elecciones menores, pero no aquellas que los devuelven al rol político-social del que están excluidos y que es un elemento central dentro del modelo de SMC (Perron et al., 2010; Storm \& Edwards, 2013). Las políticas sanitarias actuales, siguiendo la orientación de las políticas sociales en Chile, durante las últimas décadas, incorporan discursivamente la valoración de la autonomía, los derechos y la participación (Alfaro, 2004), sin embargo, estos aspectos quedan reducidos a estrategias puntuales como son la promoción de agrupaciones de familiares y usuarios de salud mental a nivel nacional (Funk, Minoletti, Drew, Taylor, \& Saraceno, 2005), pero no logran calar en el quehacer cotidiano de los dispositivos residenciales. En esta línea, la participación alcanza solo los primeros niveles del continuum de Hickey y Kipping (1998), informar y consultar, pero no logra llegar a la cogestión y al control en las decisiones. Este resultado refleja la disincronía entre los discursos y la práctica de participación en salud mental (Bang, 2014). Por tanto, se refuerza lo planteado por Méndez y Vanegas (2010), quienes señalan que desde la política sanitara se ha instrumentalizado la participación social como una forma de mejorar la salud de la población a través del uso eficiente de recursos. Al parecer la SMC termina supeditándose a un modelo biomédico que continua posicionado como un saber omnipotente que rige la vida de los que son sus objetos de intervención (Carrasco \& Yuing, 2014).

Respecto a la predicción de la restrictividad solo tres variables fueron significativas: tiempo de funcionamiento (entre uno y dos años), frecuencia de la asesoría técnica (cada 3 o 4 meses) y funcionamiento homogéneo de los dispositivos dentro del área sanitaria. Si un hogar/residencia lleva menos tiempo en funciones, tiene un menor nivel de restrictividad.
Esto parece indicar que no solo se intitucionalizan los residentes dentro de un espacio residencial, sino que también los dispositivos son institucionalizados, se puede sugerir que aquellos que poseen menos tiempo de funcionamiento, desconocen o no han adquirido la "normativa cultural" que se espera posean. En este mismo sentido, cuando los servicios se comportan como instituciones totales y no se adaptan a las necesidades de las personas, sino que funcionan de manera homogénea, el nivel de restrictividad es mayor. Esta forma de construcción social permea la subjetividad de las personas y de esta manera reproduce un sistema donde los sufrientes se transforman en individuos pasivos y alienados (Galende, 2008).

Respecto a la variable asesoría técnica, destaca que entre menos regular es esta mayor es el nivel de restrictividad del dispositivo. Esta actividad consiste en una supervisión por profesionales que dependen del programa de salud mental de las respectivas áreas sanitarias. Su objetivo está orientado a que se cumpla con la normativa ministerial (Ministerio de Salud, 2000); por tanto, este resultado podría indicar que de alguna forma el personal que realiza esta tarea estaría promoviendo un ambiente de mayor autonomía en el lugar. En consecuencia, aunque la política de salud mental en Chile ha logrado instalar un modelo de red de servicios con una serie de regulaciones basadas en principios comunitarios, a nivel microsocial es necesario trabajar en bajar estos lineamientos a las prácticas concretas de los cuidadores en los espacios residenciales donde a través de procesos intersubjetivos se promueven o limitan las libertadas de los sujetos.

En esta línea, la evaluación de las prácticas restrictivas puede considerarse como una medida de la calidad de la atención prestada en los dispositivos residenciales; esta perspectiva podría contribuir a monitorear los procesos de implementación y adecuación de las intervenciones al modelo de salud mental comunitaria. Esto facilitaría la puesta en práctica del modelo en el ejercicio cotidiano de la atención sanitaria.

La principal limitación de esta investigación es lo reducido de la muestra de hogares y residencias protegidas y el haber entrevistado a un solo cuida- 
dor por dispositivo. Sería necesario poder ampliar estos resultados con una muestra mayor.

Por otra parte, sería interesante poder contrastar estos resultados con la visión que poseen los propios residentes del hogar/residencia; para ello, se podría trabajar bajo un modelo de investigación basada en la participación de la comunidad que promueva durante el proceso los principios del modelo de SMC que se desean potenciar (Stacciarini, Shattell, Coady, \& Wiens, 2011).

\section{Referencias}

Alfaro, J. (2004). Psicología comunitaria y políticas sociales: análisis de su desarrollo en Chile. Revista de Psicología, 1(1-2), 38-54.

Bang, C. (2014). Estrategias comunitarias en promoción de salud mental: construyendo una trama conceptual para el abordaje de problemáticas psicosociales complejas. Psicoperspectivas, 13(2), 109-120.

Cantera, L. (2004). Psicología comunitaria de la salud. En G. Musitu, J. Herrero, L. Cantera \& M. Montenegro (Eds.), Psicología comunitaria y bienestar social (pp. 71-96). Barcelona: Editorial UCO.

Carrasco, J., \& Yuing, T. (2014). Lo biomédico, lo clínico y lo comunitario: interfaces en la producción de subjetividad. Psicoperspectivas, 13(2), 98-108.

Corrigan, P. (2002). Empowerment and serious mental illness: Treatment partnerships and community opportunities. Psychiatric Quaterly, 73(3), 217-229.

Funk, M., Minoletti, A., Drew, N., Taylor, J., \& Saraceno, B. (2005). Advocacy for mental health. Roles for consumers and family organizations and governments. Health Promotion International, 21(1), 70-75.

Galende, E. (2008). Lugar y destino de la subjetividad en salud mental. En E. Galende, Psicofármacos y salud mental. La ilusión de no ser (Cap. 3). Buenos Aires: Lugar Editorial.

Hickey, G., \& Kipping, C. (1998). Exploring de concept of user involvement in mental health through a participation continuum. Journal of Clinical Nursing, 7(1), 83-88.

Jordá, E., \& Espinosa, J. (1990). El proceso de desinstitucionalización en la provincia de Valencia: estudio de los alojamientos utilizados mediante la escala de prácticas restrictivas. Revista de Psiquiatría Facultad de Medicina de Barcelona, 17(2), 77-87.

Kelly, D. B. (2006). The power gap: Freedom, power and mental illness. Social Science and Medicine, 63, 1118-1128.

Kloos, B., \& Shah, S. (2009). A social ecological approach to investigating relationships between housing and adaptative functioning for persons with serious mental illness. American Journal of Community Psychology, 44(3-4), 316-326.

Kyle, T., \& Dunn, J. (2008). Effects of housing circumstances on health, quality of life and healthcare use for people with severe mental illness: A review. Health and Social Care in Community, 16(1), 1-15.

Marcos, A., \& Topa, G. (2012). Salud mental comunitaria. Madrid: Editorial UNED.

Méndez, C., \& Vanegas, J. (2010). La participación social en salud: el desafío de Chile. Revista Panamericana de Salud Pública, 27(2), 144-148.

Ministerio de Salud. (2000). Norma Técnica sobre Hogares Protegidos. Santiago, Chile: Autor.

Ministerio de Salud. (2001). Plan Nacional de Salud Mental y Psiquiatría (2.ed.). Santiago, Chile: Autor.

Ministerio de Salud. (2008). Norma Técnica para la implementación y funcionamiento de Centros de Salud Mental Comunitaria. Santiago, Chile: Autor.

Montero, M. (2009). El fortalecimiento en las comunidades, sus dificultades y alcances. Universitas Psychologica, 8(3), 615-626.

Liggins, J., \& Hatcher, S. (2005). Stigma toward the mentally ill in the general hospital: A qualitative study. General Hospital Psychiatry, 27(5), 359-364.

López, M., Fernández, L., García-Cubillana, P., Moreno, B., Jimeno, V., \& Laviana, M. (2005). Evaluación del programa residencial para personas con trastorno mental severo en Andalucía (III): características socio demográficas, clínicas y de uso de servicios de los residentes. Rehabilitación Psicosocial, 2(1), 28-39.

López, M., García-Cubillana, P., Fernández, L., Laviana, M., Maestro, J. C., \& Moreno, B. (2005). Evaluación del Programa residencial para personas con trastorno mental severo en Andalucía (II): características de los dispositivos residenciales. Rehabilitación Psicosocial, 2(1), 16-27. 
López, M., Lara, L., Laviana, M., Fernández, L., García-Cubillana, P., \& López, A. (2004). Los programas residenciales para personas con trastorno mental severo. Revisión y propuestas. Archivos de Psiquiatría, 67(2), 101-128.

López, M., \& Laviana, M. (2007). Rehabilitación, apoyo social y atención comunitaria a personas con trastorno mental grave. Propuestas desde Andalucía. Revista Asociación Española de Neuropsiquiatría, 27(9), 187-223.

Organización Mundial de la Salud. (2006). Políticas, planes y programas de salud mental. Ginebra: Autor.

Organización Panamericana de la Salud. (1991). Reestructuración de la atención psiquiátrica: bases conceptuales y guías para su implementación. Washington, DC: Autor.

Perron, A., Rudge, T., \& Holmes, D. (2010). Citizen minds, citizen bodies: the citizenship experience and the government of mentally ill persons. Nursing Philosophy, 11(2), 100-111.

Saraceno, B. (2003). La rehabilitación como ciudadanía. En B. Saraceno, La liberación de los pacientes psiquiátricos (pp. 83-109). México: Editorial Pax.

Silva, C., \& Martínez, M. (2004). Empoderamiento: proceso, nivel y contexto. Psykhe, 13(1), 29-39.

Sylvestre, J., Nelson, G., Sabloff, A., \& Peddle, S. (2007). Housing for people with serious mental illness: A comparison of values an research. American Journal of Community Psychology, 40, 125-137.

Sprioli, N., \& Silva, M. (2011). Cuidar en tiempos nuevos: el trabajo de cuidadores con pacientes psiquiátricos en residencias terapéuticas. Revista Latinoamericana de Enfermería, 19(5), 1-8.
Stacciarini, J. M., Shattell, M., Coady, M., \& Wiens, B. (2011). Review: Community-based participatory research approach to address mental health in minority populations. Community Mental Health Journal, 47(5), 489-497.

Storm, M., \& Edwards, A. (2013). Models of user involvement in the mental health context: intentions and implementation challenges. Psychiatric Quaterly, 84(3), 313-327.

Rose, D., Thornicroft, G., Pinfold, V., \& Kassam, A. (2007). 250 labels used to stigmatise people with mental illness. Health Services Research, 7, 7-97. doi:10.1186/1472-6963-7-97

Tapia, C. (2014). Relación entre el funcionamiento de un hogar/residencia protegida para personas con trastorno mental severo, el apoyo social del cuidador y las habilidades de la vida diaria de los residentes (Tesis de Magíster). Universidad de Concepción, Concepción.

Wright, P., \& Kloos, B. (2007). Housing environment and mental health outcomes: A levels of analysis perspective. Journal of Environmental Psychology, 27(1), 79-89.

Wong, Y., \& Solomon, P. (2002). Community integration of persons with psychiatric disabilities in supportive independent housing: A conceptual model and methodological considerations. Mental Health Services Research, 4(1), 13-28.

Wong, Y., Filoromo, M., \& Tennille, J. (2007). From principles to practice: A study of implementation of supported housing for psychiatric consumers. Administration and Policy in Mental Health and Mental Health Services Research, 34(1), 13-28. 
